\title{
Bilateral Giant Axillary Artery Aneurysms with Left Complete Embolism in Intravenous Immunoglobulin-Sensitive Kawasaki Disease: A Case Report
}

\section{Chen Chu}

Children's Hospital of Fudan University https://orcid.org/0000-0003-1916-9511

\section{Lan He}

Children's Hospital of Fudan University

Yi-xiang Lin

Children's Hospital of Fudan University

Fang Liu ( $\square$ liufang@fudan.edu.cn )

Children's Hospital of Fudan University

\section{Case Report}

Keywords: Kawasaki disease, systemic artery aneurysm, embolism, coronary artery aneurysm, antithrombotic therapy

Posted Date: March 30th, 2021

DOl: https://doi.org/10.21203/rs.3.rs-361296/v1

License: (c) (i) This work is licensed under a Creative Commons Attribution 4.0 International License. Read Full License 


\section{Abstract}

Background: Kawasaki disease (KD) is a systemic vasculitis that predominantly affects medium-sized arteries. In addition to well-known coronary artery aneurysms (CAAs), peripheral systemic artery aneurysms (SAAs) have also been sporadically reported. In the literatures, SAAs occurred mainly in untreated, intravenous immunoglobin (IVIG)-resistant, or severe refractory KD, and thrombotic events in SAA were rarely reported.

Case presentation: A 10-month-old boy with history of KD was referred to our hospital for suspected pseudoaneurysm of the axillary arteries. Four months prior to presentation, he had recurrent fever, conjunctival congestion, and rash. On the 10th day of fever echocardiography showed biliteral CAAs. He was then diagnosed with KD and given IVIG $2 \mathrm{~g} / \mathrm{kg}$ and aspirin at a local hospital. His fever and symptoms soon subsided and he was discharged with low dose aspirin and dipyridamole. One month prior to presentation, his parents incidentally palpated swellings in his axillae bilaterally. On admission, physical examination revealed a pulsatile swelling in his right axilla and a non-pulsatile swelling in the left. His left brachial and radial pulses were not palpable, while the pulses of other three limbs were normal. His left upper limb was cooler and less active compared to the right. Ultrasound examination revealed bilateral giant axillary artery aneurysms (AAAs) with massive thrombus in the left. Angiography confirmed bilateral giant AAAs with left AAAs completely embolized and fine collateral vessels connecting to the distal brachial artery, in addition to bilateral multiple giant CAAs without stenoses. The patient was given intravenous prostaglandin for ten days to allow for formation of collateral circulation, as well as aspirin, low molecular weight heparin (which was switched to warfarin before discharge) and metoprolol. At discharge, the temperature and movement of his left upper limb improved significantly. On follow-up at three months, his left limb improved and was similar to the right with no cardiovascular event having occurred. The images of CAAs and AAAs on ultrasound and computerized tomography remained the same.

Conclusions: This case highlights the importance of evaluating peripheral SAAs in KD patients with CAA, even if their clinical course appears uncomplicated. For both large non-aortic SAAs and CAAs in KD patients, antithrombotic therapy is of utmost importance.

\section{Background}

Kawasaki disease (KD) is a systemic vasculitis that predominantly affects medium-sized arteries. In addition to well-described coronary artery aneurysms (CAAs), it also can involve non-coronary systemic arteries (1). The incidence of systemic artery aneurysms (SAAs) by angiography in untreated KD children has been reported to be approximately $2 \%$ (2). In a recent study conducted by our team, the incidence of SAAs was also estimated to be $2 \%$ among all KD patients (3). Previous literature (2-9), predominantly case reports, revealed that SAAs in KD patients were generally associated with coronary artery lesions, especially giant CAAs, and were more likely to occur in infants and patients with a high risk of developing CAAs, including untreated KD and IVIG-resistant or severe refractory KD. In addition, thrombotic events 
relating to SAAs have rarely been reported. Here we report an intravenous immunoglobulin (IVIG)-sensitive $\mathrm{KD}$ case who developed both giant CAAs and bilateral giant axillary artery aneurysms with left complete embolism.

\section{Case Presentation}

A 10-month-old boy was referred to our hospital because swellings were found in his bilateral axillae. Four months prior to referral, the infant had recurrent fever, followed by bilateral conjunctival congestion and a maculopapular rash on his limbs and trunk. Laboratory tests showed elevated leucocytes and C reactive protein. His fever persisted after antibiotic therapy. On the 10th day of fever, echocardiographic examination at a local hospital showed dilation of bilateral coronary arteries (there were no documented diameters for each coronary artery). He was then diagnosed with Kawasaki disease (KD) and administrated intravenous immunoglobulin (IVIG, 2g/kg/dose) and aspirin (40mg/kg/d q8h) immediately. After finishing the IVIG infusion, his fever subsided and clinical symptoms and laboratory test improved uneventfully. Three days later, he was discharged with low dose aspirin and dipyridamole. The infant was followed up at the local hospital due to restrictions relating to the COVID-19 pandemic, and he remained afebrile and echocardiography showed no obvious changes. One month prior to referral, his parents incidentally felt swellings in his bilateral axillae, but they did not think much of it. Three days before presentation to our hospital, the infant had an ultrasound done at the local hospital his regular follow-up, which found suspected axillary artery aneurysms or pseudoaneurysms. He was then immediately referred to our hospital for further evaluation.

On admission, a pulsatile swelling in the right axilla and a non-pulsatile swelling in the left axilla were palpated. The left brachial and radial pulses were impalpable. The skin temperature of the left upper limb was lower than that of the right one, and the left palm was pale. The movement of left upper limb was less active than that of the right. The pulses, skin temperature, and color of right upper limb and both lower extremities were all normal.

After admission, ultrasound showed a giant right axillary artery aneurysm (AAA) $26 * 12.7 * 19.5 \mathrm{~mm}$ in size with a wall thickness $1.2 \mathrm{~mm}$. The flow inside the aneurysm was patent with no stenosis and the diameters of the distal and proximal adjacent arteries were about 2.3-3.0mm (Figs. 1a \& b). In left axilla, a large heterogeneous mass $(41.4 * 11.7 * 15.8 \mathrm{~mm})$ without blood flow was detected which suggested complete occlusion (Figs. 1c \& d).

No abnormalities were found on echocardiography except for bilateral multiple giant coronary artery aneurysms (CAAs) involving the left anterior descending artery (LAD) and right coronary artery (RCA). The internal diameters of the aneurysm in the LAD, the proximal RCA, and mid RCA were $7.48 \mathrm{~mm}$ ( $\mathrm{z}$ score $=+$ 18.65), $9.0 \mathrm{~mm}(z$ score $=+20.76)$ and $5.09 \mathrm{~mm}(z$ score $=+10.64)$ respectively. Investigations to rule out myocardial ischemia including 12-lead electrocardiography, 24-hour Holter monitor, 2-dimention and tissue Doppler echocardiography, and ATP stressed nuclide myocardial perfusion imaging were all normal. 
Angiography was done for the patient. Coronary angiography showed bilateral multiple giant CAA, including a $6.0 * 8.5 \mathrm{~mm}$ CAA at the bifurcation of the LAD and circumflex branch (Fig. 2a) and several beaded CAAs in RCA from proximal to the origin of the posterior descending coronary artery (the diameters of aneurysms were $9.0 \mathrm{~mm}, 7.6 \mathrm{~m} \mathrm{~m}, 6.8 \mathrm{~mm}$, and $5.2 \mathrm{~mm}$, respectively) (Fig. 2b). An incomplete thrombus could be seen in the third aneurysm of the RCA (Fig. 2b, arrows). Fortunately, the flow of the LCA and RCA was patent with no stenoses or occlusions. Left and right subclavian arteriography showed bilateral giant AAAs. The size of the right AAA was $15.5 * 25.0 \mathrm{~mm}$ with no thrombus in the aneurysm (Fig. 2c). The blood flow throughout the right upper limb was normal. The size of left AAA was $16.5 \star 45.0 \mathrm{~mm}$, with complete embolism inside the aneurysm. There were some fine collateral vessels connecting to the distal brachial artery, and the blood flow into the distal left upper limb was slow and sparse (Fig. 2d). No abnormalities of other peripheral arteries were found.

After completing investigations and consulting with the vascular surgical team, we administrated intravenous prostaglandin for this baby to accelerate angiogenesis of the collateral vessels instead of surgical repair of the occluded aneurysm. At the same time, oral aspirin $5 \mathrm{mg} / \mathrm{kg}$ per day and low molecular weight heparin $(150 \mathrm{u} / \mathrm{kg} /$ dose $\mathrm{q} 12 \mathrm{~h})$ were given to prevent thrombosis in the right AAA and CAAs. Metoprolol was used to reduce myocardial oxygen demand because of the giant CAAs. After ten days of treatment, the patient's skin temperature and movement of left upper limb had improved, and a weak pulse of left brachial artery could be palpable. Low molecular weight heparin was then switched to warfarin, with international normalized ratio (INR) monitored to be near 2.5.

Three months after discharge, the patient was followed up. During his stay at home, he was asymptomatic and grew well. Physical examination showed that skin temperature, movement, and left upper limb pulses further improved to a similar level to that of the right side. CT angiography confirmed that the blood flow of the brachial artery at the distal part of the embolized left axillary artery was significantly increased (Fig. 2e). Repeat echocardiography showed the coronary arteries remained the same as before; electrocardiography was normal.

\section{Discussion}

In addition to CAAs commonly seen in KD, non-coronary systemic artery aneurysms can also be a sequelae of KD. In previous reports on SAAs in KD, SAAs were more likely to occur in patients with a high risk of developing CAAs, including untreated KD, IVIG-resistant, or severe refractory KD. In this case, although there was somewhat of a delay in the diagnosis and treatment of the patient, on the 10th day of onset, there was response to one dose of IVIG. However, his CAAs occurred before treatment, and it is estimated that his bilateral axillary aneurysms were already present at the same time.

In addition to systemic aneurysms caused by vasculitis, peripheral aneurysms in children can also be caused by idiopathic, traumatic, infectious, and other causes. If these aneurysms are large or cause symptoms, surgery is recommended considering the risk of rupture (10). However, there is no consensus on the treatment of systemic aneurysms in children with KD. Previous studies on the prognosis of SAAs 
in $\mathrm{KD}$ showed that $80 \%$ of SAAs could regress to normal within 6 months, and a small number of aneurysms could stenose, but rupture had never been reported $(2,3,11)$. In addition, the systemic aneurysms of KD mainly occurred in medium-sized arteries rather than the aorta. Therefore, there is no high risk of rupture even for large SAAs. Similarly, ruptures of large coronary aneurysms secondary to KD have rarely been reported and usually occur in the acute phase (1). Therefore, for large non-aortic aneurysms secondary to KD, more attention should be paid to the risk of thrombosis and embolism rather than rupture. In previous cases, surgical or interventional treatment of aneurysms has been reported only in few cases. Two KD patients separately complicated with giant aortic arch aneurysms and thoracoabdominal aortic aneurysms underwent aneurysm resection and aortic reconstruction with artificial conduits considering the risk of rupture $(12,13)$. Another two cases of KD separately with hepatic and intercostal aneurysms were treated with percutaneous coil embolization $(14,15)$. In view of the pathological characteristics of Kawasaki disease vasculitis, the main treatment of large SAAs is antithrombotic therapy, which is the same as that of CAAs, that is, large SAAs need an anticoagulation regimen of both aspirin and warfarin. In this case, giant axillary aneurysms and CAAs were not detected in time at the local hospital, and only aspirin was given for anti-thrombosis, resulting in complete occlusion of the left AAA and associated ischemic symptoms. Considering that the infant's blood vessels are small in diameter and collateral vessels had formed, vascular surgeons suggested vasodilators be given to let the collateral circulation further develop instead of intervening with surgical bypass, and adequate antithrombotic treatment was given to maintain patency of the giant right AAA and multiple large CAAs. The right AAA was located in the axilla, which is relatively hidden, so the risk of rupture caused by external force is very small. However, parents have been advised not to hold the baby with their hands in these areas. Follow-up examinations confirmed that the status of the right large AAA was stable, and the distal blood supply of the left upper limb had further improved.

\section{Conclusion}

This case highlights the importance of evaluating peripheral SAAs in KD patients who are treated relatively late or have initial coronary artery involvement, even if patients are sensitive to IVIG treatment. For large non-aortic SAAs in KD patients, the risk of embolism should be given precedence over concerns of aneurysm rupture.

\section{Abbreviations}

KD: Kawasaki disease; CAAs: Coronary artery aneurysms; IVIG: Intravenous

immunoglobulin; SAAs: Systemic artery aneurysms; AAAs: axillary artery aneurysms; LAD: left anterior descending artery; RCA: right coronary artery

\section{Declarations}

Acknowledgments 
We thank Conway Niu from the Royal Aberdeen Children's Hospital for his kind English language consultation.

\section{Authors' contributions}

LF directed the management of the patient and assisted in drafting, revising, and reviewing the manuscript; CC managed the patient and was a major contributor in writing the manuscript; $\mathrm{HL}$ and LYX assisted in the acquisition of the initial data. All authors read and approved the final manuscript.

\section{Funding}

This study was supported by the National Natural Science Foundation of China (82070513), Shanghai Natural Science Foundation (20ZR1408500), and Young Clinical Scientist Program, National Children's Medical Center (EK112520180309).

\section{Ethics approval and consent to participate}

Not applicable.

\section{Consent for publication}

Consent has been given by the parents.

\section{Availability of data and materials}

Not applicable.

\section{Competing interests}

The authors declare that they have no competing interests.

\section{References}

1. McCrindle BW, Rowley AH, Newburger JW, Burns JC, Bolger AF, Gewitz M, et al. Diagnosis, Treatment, and Long-Term Management of Kawasaki Disease: A Scientific Statement for Health Professionals From the American Heart Association. Circulation. 2017;135(17):e927-e99.

2. Kato $H$, Sugimura T, Akagi T, Sato N, Hashino K, Maeno $\mathrm{Y}$, et al. Long-term consequences of Kawasaki disease. A 10- to 21-year follow-up study of 594 patients. Circulation. 1996;94(6):137985.

3. Zhao QM, Chu C, Wu L, Liang XC, Sun SN, He L, et al. Systemic Artery Aneurysms and Kawasaki Disease. Pediatrics. 2019;144(6).

4. Cabrera ND, Sridhar A, Chessa M, Carminati M. Giant coronary and systemic aneurysms of Kawasaki disease in an infant. Pediatr Cardiol. 2010;31(6):915-6. 
5. Heran MK, Hockley A. Multiple mirror-image peripheral arterial aneurysms in Kawasaki disease. Pediatr Cardiol. 2011;32(5):670-3.

6. Ekici F, Varan B, Kocabas A, Erdogan I, Eminoglu S, Aktas D. Multiple giant aneurysms and stenoses of the coronary and systemic arteries in an infant with kawasaki disease at the early stage of convalescent period. Echocardiography. 2014;31(5):E147-50.

7. Johnston N, Coleman D, McMahon CJ. Extensive coronary and systemic arterial aneurysm development in severe refractory Kawasaki disease. Cardiol Young. 2017;27(2):375-6.

8. Roy S, Biswas MK. Multiple Systemic Aneurysms in a Case of Neglected Kawasaki Disease. J Paediatr Child Health. 2019;55(1):117.

9. Musa H, Yubbu P, Koh GT. Internal iliac artery thrombosis in a 2-month-old infant with incomplete Kawasaki disease. Cardiol Young. 2020;30(1):142-4.

10. Hall HA, Minc S, Babrowski T. Peripheral artery aneurysm. Surg Clin North Am. 2013;93(4):911-23. ix.

11. Hoshino S, Tsuda E, Yamada O. Characteristics and Fate of Systemic Artery Aneurysm after Kawasaki Disease. J Pediatr. 2015;167(1):108-12. e1-2.

12. Petrunic M, Drinkovic N, Stern-Padovan R, Mestrovic T, Lovric D. Thoracoabdominal and coronary arterial aneurysms in a young man with a history of Kawasaki disease. J Vasc Surg. 2009;50(5):1173-6.

13. Hakim K, Boussada R, Chaker L, Ouarda F. Giant aortic arch aneurysm complicating Kawasaki's disease. Ann Pediatr Cardiol. 2014;7(3):201-3.

14. Neuwirth CA, Singh H. Intercostal artery aneurysm in a child with Kawasaki disease and known coronary artery aneurysms. J Vasc Interv Radiol. 2010;21(6):952-3.

15. Paolantonio G, Tarissi de Jacobis I, Marchesi A, Natali GL, de Ville de Goyet J, Rollo M, et al. Right hepatic artery aneurysms in a child with Kawasaki disease: flow-preserving endovascular treatment. Ann Hepatol. 2014;13(2):284-7.

\section{Figures}



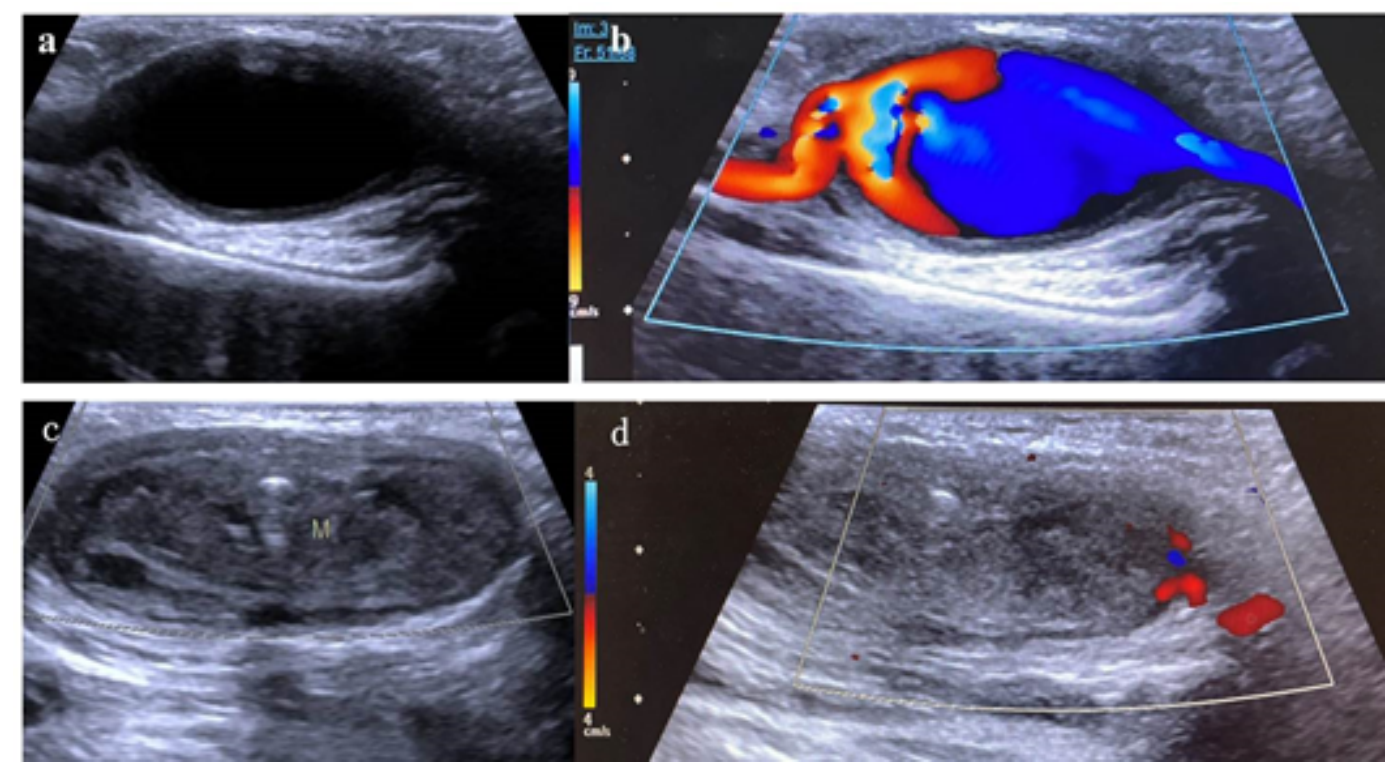

\section{Figure 1}

The ultrasound images of bilateral axillary artery aneurysm. a-b. The right giant aneurysm (a, without color Doppler; b, with color Doppler) without thrombus with the flow velocity of input and output site of the aneurysm $0.8 \mathrm{~m} / \mathrm{s}$ and $0.6 \mathrm{~m} / \mathrm{s}$ respectively; c-d. The left giant aneurysm with massive thrombus occlusion (c, without color Doppler; $d$, with color Doppler) and sparse blood flow near the mass.

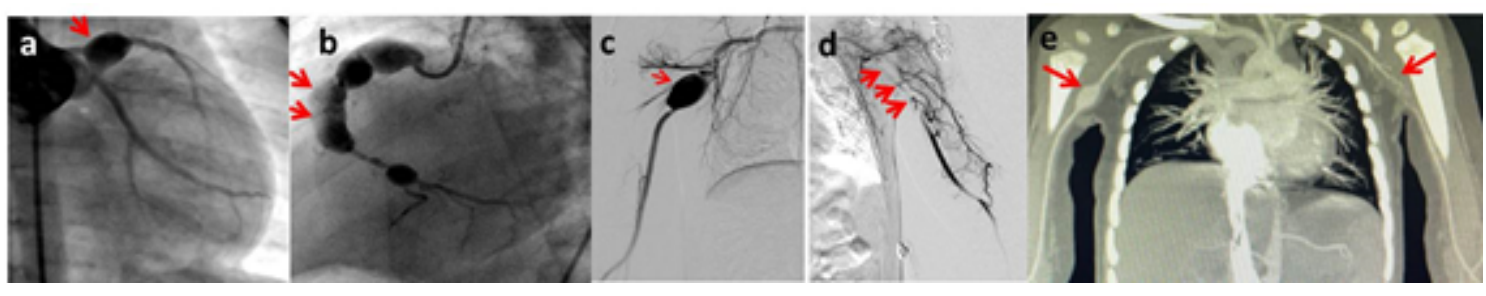

\section{Figure 2}

Angiographic images showing a. A 6.0*8.5mm aneurysm (arrow) at the bifurcation of the left anterior descending artery and circumflex branch. b. Beaded aneurysms in the RCA from proximal branch to the bifurcation of the posterior descending coronary artery. An incomplete thrombus can be seen in the third aneurysm (arrows). c. Right giant axillary artery aneurysm (arrow) with normal blood flow. d. Left giant AAA which has been completely embolized (arrows) with fine collateral vessels supplying blood to the distal brachial artery. e. More blood flow to the left brachial artery distal to the occluded AAA on CT angiogram at 3-month follow-up compared to that of 3 months prior (d). 